\title{
Médiévales
}

Langues, Textes, Histoire

47 | automne 2004

Îles du Moyen Âge

\section{Îles et tissu « connectif » de la Méditerranée médiévale}

\section{Henri Bresc}

\section{(2) OpenEdition}

1 Journals

\section{Édition électronique}

URL : https://journals.openedition.org/medievales/823

DOI : $10.4000 /$ medievales.823

ISSN : $1777-5892$

\section{Éditeur}

Presses universitaires de Vincennes

\section{Édition imprimée}

Date de publication : 1 décembre 2004

Pagination : 123-138

ISBN : 2-84292-157-7

ISSN : 0751-2708

\section{Référence électronique}

Henri Bresc, « Îles et tissu « connectif » de la Méditerranée médiévale », Médiévales [En ligne], 47 | automne 2004, mis en ligne le 02 septembre 2006, consulté le 24 avril 2022. URL : http:// journals.openedition.org/medievales/823; DOI : https://doi.org/10.4000/medievales.823

Ce document a été généré automatiquement le 24 avril 2022.

Tous droits réservés 


\title{
Îles et tissu « connectif » de la Méditerranée médiévale
}

\author{
Henri Bresc
}

1 Dans leur récent ouvrage ${ }^{1}$, Peregrine Horden et Nicholas Purcell réservent aux îles de la Méditerranée un traitement particulier. Il s'agit pour eux de démontrer, ou au moins de suggérer, la relative permanence de la "connectivité » maritime pendant toute l'Antiquité et au Moyen Âge. Elle connaît certes une flexion aux hautes époques, mais elle retrouve vite, selon eux, des modèles pérennes. La Méditerranée est en effet décrite comme une mosaïque de niches écologiques aux économies changeantes dictées par des stratégies de marché, par la saisie puis l'abandon de cultures opportunistes, et permises par la primauté de la mer, des transports rapides, massifs, économiques, sans obstacles, sinon sans risques. L'ouvrage, qui s'inspire largement, mais pas toujours explicitement, de Fernand Braudel ${ }^{2}$, se veut aussi un « défi à Braudel », comme l'a relevé Brent D. Shaw dans un compte rendu remarquable ${ }^{3}$, qui insiste aussi sur la présence dans la Méditerranée de réponses adéquates à plusieurs questions que posent Horden et Purcell. On trouvera dans cette Corrupting Sea, au titre encore mystérieux, un peu de polémique, d'immenses lectures, quelquefois hâtives (les auteurs font régulièrement dire au signataire de ces lignes le contraire de ce qu'il écrit sur la Sicile, mais l'équivalent de ce que pense un de leurs collègues britanniques), un ensemble de citations et de résumés fort utiles que l'on a qualifié astucieusement de "boîte à outils ", et une infinité de bonnes remarques et de réflexions que l'on peut prolonger. Le thème de la " connectivité » unit tout cela sans emporter la conviction du médiéviste : il existe en effet des temps où la Méditerranée est une mer morte et des espaces où la guerre navale, facile à organiser, rapide à l'attaque, aisée à concentrer, n'a apporté que destructions et repli continental. Nous le verrons plus loin.

Quelle est donc la part des îles dans le modèle dessiné par Horden et Purcell? La primauté des transports maritimes leur donne une fonction très précoce de portail de la terre ferme, dès le II ${ }^{\mathrm{e}}$ millénaire avant l'ère. Plus généralement, la terre ferme ellemême peut s'analyser comme un archipel de niches, tandis que les points remarquables de la côte voient s'établir des poches de contrôle fortifiées vers le continent et 
ravitaillées par la mer. Péninsules barrées, châteaux comme Bodrum, ports bien protégés alternent avec des dominations pacifiques: Antiphellos, en face de l'île de Castellorizo/Mégistè, est sur la côte de l'Anatolie, la vigne des insulaires et, à $9 \mathrm{~km}$ de Ceuta, quasi-île vouée à être le centre des communications du Détroit de Gibraltar, Balyûnash est aussi la vigne et le jardin des habitants du port quasi-insulaire.

3 La petite île est d'autre part vouée à la spécialisation agricole et minière, si la géologie et l'hydrographie le permettent: vins des îles de l'Égée antique, raisin sec des îles Ioniennes modernes, mastic de Chios et autres lieux, marbres, obsidienne, métaux, selon les îles, terre de Lemnos correspondent à des niches d'intensification ou à des activités extractives aisément tournées vers une exportation commode. Les péninsules sont d'autres îles, en ce sens, comme celle de Phocée. Pour toutes, suivant le géographe E. Kolodny ${ }^{4}$, Horden et Purcell insistent sur l'instabilité et la vulnérabilité de l'implantation humaine. L'exemple de Mélos/Milo, d'une surface de $150 \mathrm{~km}^{2}$, mais dont $80 \%$ sont inutilisables en raison d'une formidable érosion entamée par le déboisement à l'Âge du Bronze, est typique des difficultés et des défis de l'économie insulaire : on a exploité l'obsidienne, puis tenté des cultures en terrasses, mais, contrairement à ce qu'implique la fière réponse aux Athéniens, l'île n'a jamais connu d'autarcie tranquille.

4 Une troisième remarque, p. 351, souligne que les îles ont souvent une population nombreuse: Naxos fournit 8000 hoplites au $V^{e}$ siècle, Minorque et Majorque hellénistiques compteraient chacune 30000 habitants. Les deux historiens britanniques comparent ces chiffres à ceux du XIX ${ }^{e}$ siècle, ce qui relève d'une méthode moins sûre, et en déduisent que l'île trouve son soutien économique dans le commerce, comme emporium, dans la navigation, dans le mercenariat et la migration rurale temporaire. Ce que les médiévistes accepteront pour bien des échantillons, dont la Corse, pour l'émigration, Malte aussi, mais sans trop généraliser. La Sicile, la Sardaigne, durablement sous-peuplées, sont des terres d'immigration, de même que la Rhodes des Chevaliers, ou les Baléares au XIII siècle, après la reconquête

5 Un quatrième volet de la réflexion d'Horden et de Purcell conduit à la sainteté des îles. La grâce divine rattache en effet les îles, ou plutôt des îles relativement peu nombreuses, à une géographie de la mobilité, de la circulation navale des pèlerins et des simples voyageurs; cette vaste perspective repose sur quelques rochers inscrits $\mathrm{du}$ côté de la mer (Syros où l'on invoque Euploia, Aliki, au sud de Thasos, Grammata sur la terrible côte acrocéraunienne), sur les caps protégés par les temples chers à Ellen Semple $^{5}$, sur les îles saintes enfin, Poros, Délos, Patmos, Samothrace. Peut-on leur associer le Lérins, ou cette île qui fait face à la Cherson byzantine et où la tombe de saint Clément attire les pèlerins byzantins? Autant d'époques, autant de raisons différentes de cultiver la sainteté dans l'espace fermé ou ouvert, protégé ou connectif, isolé ou pénétré de commerces et de voyages, des îles.

Qu'en pense le médiéviste? La séduction du livre d'Horden et Purcell repose sur le court-circuit systématique des époques; on se sent intelligent à trouver des éléments qui confortent les auteurs dans ce mouvement perpétuel, « brownien », qui agite la mer et bénit ses rives et ses îles. Mais il faut raison garder et distinguer des mers utilisées, occupées, même par des malfaisants, et des mers vides, des temps vides, abandonnés. On acceptera volontiers l'analyse qui voit des habitats disproportionnés avec les ressources propres des îles à la fin de l'époque antique : à Pantelleria, par exemple, à mi-chemin entre la Sicile et le Cap Bon, la ville byzantine paraît très vaste, elle accueille les réfugiés de Carthage après la chute de la Tunisie aux mains des armées musulmanes. 
Peut-être le port était-il alors mieux protégé qu'aujourd'hui : il pouvait héberger les dromons. Peut-être s'agissait-il d'une garnison ravitaillée de Sicile, puis directement d'Orient... Mais l'île est ensuite prise et laissée quelque temps à l'abandon, pour être repeuplée par des musulmans sans doute au $\mathrm{XI}^{\mathrm{e}}$ siècle ; aride, sans aucune source, elle n'avait d'intérêt que comme point d'observation sur le passage des flottes adverses, ou base d'offensive. Sa fonction au service de la flotte byzantine n'est retrouvée que quand la mer est divisée, et que les flottes pisanes, puis normandes de Sicile, menacent l'Ifrîqiyya. Alors, l'île redevient la sonnette d'alarme qui protège Mahdiyya et Sousse, grâce aux pigeons voyageurs, les « messagers volants de l'Islam ».

7 J'envisagerai donc successivement les quatre points évoqués ci-dessus en m'appuyant principalement sur la documentation sicilienne, qui éclaire la grande île et ses annexes, les archipels des Égades, des îles éoliennes, de Malte, Comino et Gozo, et Pantelleria l'isolée ${ }^{6}$. Et j'envisagerai pour finir un modèle de « connectivité » qu'Horden et Purcell réservent sans doute pour le second volume, annoncé, à savoir la stratégie navale proprement militaire. Mais, dans tous les cas, j'éviterai de valoriser le cabotage, les rapports à courte distance, le tissu connectif du voisinage. La Méditerranée est d'abord, au Moyen Âge, un ensemble de mers étroites, mais aux rives rarement complémentaires. Il faut donc aller chercher les produits et porter les hommes fort loin : dès les premiers documents de la Geniza, la Sicile est au cœur d'une relation entre Alméria et Alexandrie. Les îles petites et moyennes y perdent de leur intérêt : le modèle n'est pas celui d'un échange incessant entre niches écologiques voisines et complémentaires.

Les temps de la mer morte et ceux de la mer partagée

8 La mer n'est pas toujours cet espace ambigu sur lequel les cités classiques ouvrent une façade, même quand elles n'y ont pas de port, comme la colline des temples d'Akragas qui domine la plage de San Leone. Les mers byzantines, par exemple, ont connu d'abord un vif mouvement de retrait des habitats insulaires, pendant la domination de l'émirat musulman de Crète, l'« Arabocratie ", de 827 à 961, puis une réoccupation au XI ${ }^{e}$ et au XII siècle, quand l'Égée a accueilli les réfugiés anatoliens fuyant les Turcs; de nouveaux évêchés sont alors érigés, mais les plus petites des îles restent désertes, Anticythère, Antiparos, Fourni, Psara, Délos. En 1420, Nompar de Caumont, qui a l'œil militaire, note l'abandon total des Christiani, de Sikinos, de Pholégandros, de Polyâgos, d'Antimilos, de Karavi ${ }^{7}$. Les raids des émirs turcs et les grandes expéditions ottomanes vident enfin Ténédos, Icarie et même Samos, au témoignage de Belon du Mans en 1546. Mais il y a plus fort, au cœur même de l'espace méditerranéen: Malte est totalement abandonnée après la destruction de la ville et de la garnison byzantine par les musulmans d'Ifrîqiyya en 869. L'archipel maltais est restée vide, au témoignage de Himyarî ${ }^{8}$, au cœur d'une mer morte, jusqu'en 1048-9, quand Malte a été recolonisée par une poignée de Maghrébins, d'où l'épisode fameux et légendaire des esclaves libérés et mariés avec les filles des colons pour résister aux Byzantins. La redécouverte de ce texte a fait l'effet d'une bombe : il implique l'interruption de la continuité apostolique et épiscopale, le christianisme paulinien de l'île est une restauration du XII siècle sur une population majoritairement musulmane, les chrétiens étant des immigrants de Sicile. On est loin ici du tissu connectif. En revanche, les enclaves terriennes d'Horden et Purcell se sont effectivement multipliées pendant ce long temps des mers mortes. Mais ce sont maintenant des quasi-îles guerrières et pillardes ravitaillées et soutenues par la mer, émirat de Bari, Garigliano, Freinet, et l'Arabocratie de l'archipel, Crète, 
Naxos, Athènes. Il faut les analyser comme des fronts de carrière pour l'extraction d'esclaves destinées à fournir les métropoles du monde musulman, Cordoue, Kairouan et Fustat. C'est l'envers guerrier d'une "connectivité » dont la survie est à peine assurée par quelques marchands amalfitains.

9 Cette situation, où les relations pacifiques atteignent leur nadir, se retrouve à partir du $\mathrm{XV}^{\mathrm{e}}$ siècle en Méditerranée centrale : les îles y perdent le rôle de relais militaire qui a été le leur au moment de la grande hégémonie normande, puis frédéricienne et enfin catalane sur le Canal de Sicile et sur les mers étroites qui séparent l'Italie du sud de l'Afrique et de la péninsule hellénique. Pantelleria, pourtant habitée par une communauté mudéjare placée quelque temps sous la double autorité du roi de Sicile et $\mathrm{du}$ calife hafside, puis purement sicilienne, perd sa fonction commerciale encore attestée vers 1370 , et se replie sur la course. Cette activité incertaine finance mal, au demeurant, une garnison trop lourde. Malte garde sans doute des relations plus ou moins licites avec Mahdiyya et Sousse, mais elle est placée maintenant in fronteria Barbarorum et glisse à son rôle moderne de base de piraterie, attirant d'effroyables représailles hafsides. La vision de Braudel contraste évidemment avec celle des auteurs britanniques puisqu'elle prend en compte cette évolution vers la «frontière ", poreuse sans doute et accueillante aux transfuges et aux renégats, mais dure, brutale. Malte et Pantelleria pourraient illustrer cette "brutalisation» des rapports humains qui est aujourd'hui à la mode historienne : un capitaine corsaire, Maxhamet lu Mineri, se fait chrétien à Pantelleria et parcourt la Sicile en vendant comme esclaves ses anciens compagnons de piraterie; un garçon né de femme maltaise captive à Sousse, mais conçu à Malte avant l'enlèvement de sa mère, part en course avec ses camarades musulmans, déserte et se fait baptiser à Pantelleria.

On retrouve donc au $\mathrm{XV}^{\mathrm{e}}$ siècle des modèles classiques du temps de la frontière islamobyzantine, l'image en particulier de saint Jean Thériste, né à Palerme d'une mère enlevée en Calabre et concubine de l'émir. Une expédition de pillage lui donne l'occasion de choisir le retour au christianisme, l'ermitage pacifique et la sainteté. Il ne faudrait pas seulement opposer des temps de relations pacifiques et des temps guerriers, mais plutôt, en ce qui concerne les îles, des temps de relations et des temps de latence, à peine parcourus de quelques expéditions. Le retrait des populations côtières et l'abandon des habitats insulaires rendent la course fort peu rentable sans un courant très actif de commerce en haute mer. La Sicile, particulièrement exposée, voit

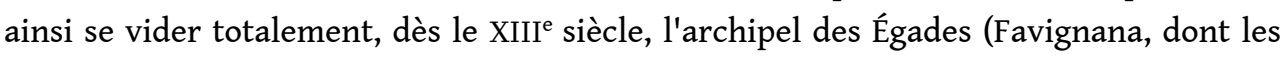
églises normandes sont attestées par les rôles des madragues, Marettimo et Levanzo), l'île d'Ustica, qui héberge encore à la fin du XIII siècle un monastère cistercien, bientôt replié à Palerme, et l'archipel éolien, à l'exception de l'île de Lipari, elle-même base corsaire napolitaine, angevine, destinée à harceler la grande île.

11 En temps de paix relative, les îles balisent les routes, permettant de « rafraîchir l'estime" après une navigation en droiture, de rectifier le cap, donc ${ }^{9}$. Comme les montagnes et les caps, elles fournissent les repères qui facilitent les atterrages, et scandent donc les récits de voyages, Formentera, Ustica, Stromboli, les Gozzes de Candie et toutes les îles de l'Archipel méridional et du Dodécannèse, Milos, Pholégandros, Santorin, Astypalia, Karpathos, Lango/Côs, Episcopi/Tèlos, Nysiros, Chalki, Symi. Le géographe sicilien al-Idrîsî, vers 1158, synthétise des portulans byzantins perdus : il met en lumière un faisceau de routes Est-Ouest, du Péloponnèse à Chypre, par Mykonos et Délos, et de l'Hellade à Samos et Chios $^{10}$. Une autre route va 
d'île en île de Constantinople à Naxos, puis à la Crète, inscrite dans le portulan militaire de Constantin VII Porphyrogénète, le Stadiodromikon. Les mouillages abrités jouent un rôle de refuge en cas de tempête, ou de bora, vent violent de nord-est de l'Adriatique : ils sont signalés avec soin par les portulans. Pero Niño, capitaine et corsaire à l'occasion, fait ainsi souvent mouillage aux îles, à Capraia, aux Alhabibas, à Zembra' ${ }^{11}$. Il faut préciser que les îles désertes ou peu peuplées servent aussi de point d'embuscade pour les guets-apens de la course, comme pour les grandes batailles navales: îles d'Hyères, Formigues, Meloria, Ponza, Ustica, Favignana, tandis que celles qui sont munies d'un château peuvent jouer le rôle de sonnettes d'alarme; c'est le cas de Pantelleria, mais aussi de Lango/Côs, de Castellorizo, fournies de "farons", feux d'avertissement, par les Hospitaliers : ils signalent les navires en variant intensité et nombre des feux ${ }^{12}$. L'île sert enfin de port de rachat : un corsaire valencien capture des Tunisiens, les force à se dire Bougiotes en leur disant que, s'ils acceptent, ils pourront payer rançon à Pantelleria où il les vendra. Sinon, il les portera en Romanie. Par chance, un marchand messinois, Barthélemy de Malte, monté à bord les acheter, les reconnait ${ }^{13}$. On identifie là la familiarité des mers étroites, mais le rôle des îles dans le tissu connectif apparaît bien ambigu.

Les spécialisations insulaires

12 L'idée d'une spécialisation agricole ou minière est séduisante, comme celle de l'emporium; on voit tout de suite la réussite génoise de Chios, où le mastic, plantation pilotée et monopole de vente, produit la moitié des revenus de l'île ou les alunières de la quasi-île de Phocée. On trouvera aisément pour le second Moyen Âge des cultures spécialisées et des industries extractives. Dans l'espace sicilien, Malte, qui ne cultive que l'orge et a besoin d'une importation régulière de céréales, compense une partie de ces achats par l'exportation du coton et du cumin. Lipari possède aussi quelques plantations de coton, de même que Pantelleria. Cette dernière n'a pas encore découvert le raisin sec et les câpres, qui assureront sa survie avec l'émigration dans la régence de Tripoli, puis dans le protectorat de Tunisie, avant que le tourisme prenne le relais. On pensera aussi aux vignes du Cap Corse, aux mines sardes, au sucre chypriote. L'économie de Majorque, au XIV siècle, repose encore sur un échange entre des productions locales, olives, huile, figues sèches, sel d'Eivissa, et le blé sarde et sicilien. L'activité commerciale à destination de l'Afrique soutient cet échange. La combinaison est étroite entre les revenus du transport, des trafics, la disponibilité continue d'une flotte et la capacité d'assurer le ravitaillement, toujours angoissant, de l'île. Mais cet archipel de spécialisations n'est en rien différent des enclaves continentales : l'olivette des Pouilles, dès le XI ${ }^{\mathrm{e}}$ siècle, la vigne des côtes tyrrhéniennes de la Calabre, de Patti et de l'Etna en Sicile, les cannes à sucre de Palerme, puis de Valence, constituent autant d'isolats engagés dans un commerce qui n'a rien de "connectif», qui est à longue distance, essentiellement maritime et pour des marchés urbains riches qui payent bien.

13 L'artisanat ou la manufacture peuvent jouer le rôle de la spécialisation: Pantelleria byzantine, dans une mer pacifiée après la destruction du royaume vandale, exporte des meules de basalte et surtout une céramique culinaire grossière, montée sans tour et riches d'inclusions, que la fouille identifie en Sicile, à Carthage, à Naples et jusqu'en Espagne. Elle importe en revanche des céramiques fines africaines, palestiniennes et syriennes qui vont avec des produits de qualité, huile et vin. Le niveau de vie élevé de la grosse agglomération sise sur l'acropole de San Marco est attesté par la qualité des mosaïques retrouvées. La production de masse, presque industrielle, des marmites en 
«Pantellerian Ware », permet sans doute de compenser une part des importations et d'insérer l'île dans un marché de redistribution autour de la mer Tyrrhénienne jusqu'alors exclusivement dominé par les céramiques africaines. La Malte de la fin du Moyen Âge connaît aussi une véritable industrie rurale, filage et tissage de cotonnades rustiques exportées en Sicile, mais aussi au-delà, dans l'aire que couvre le commerce catalan et les trafics des Ragusains : ce sont des toiles à matelas solides. Quant à Majorque, le développement de son industrie drapière relaie, fin $\mathrm{XIV}^{\mathrm{e}}$ et début $\mathrm{XV}^{\mathrm{e}}$ siècle, sur le modèle languedocien et catalan, les spécialités rurales.

Est-ce à dire que cette spécialisation est une constante ? L'étude minutieuse d'Élisabeth Malamut sur les îles byzantines repeuplées après l'Arabocratie montre la coexistence d'une économie insulaire majoritairement vivrière et de cultures d'exportation rares ${ }^{14}$. Le mastic est déjà présent à Chios, mais aussi, semble-t-il, dans d'autres îles. Le vignoble largement répandu (Chios, Mitylène, Kôs, Rhodes) est destiné au ravitaillement de la Grande Ville, Byzance. Il ne s'agit donc pas d'une économie de simple subsistance, au niveau du foyer fiscal ou de l'exploitation rurale, mais de combinaisons à l'échelle de chacune des îles pour mettre en valeur un maximum de ressources, ce qui s'accorde avec le thème principal d'Horden et Purcell. Mais en dehors d'une route des vins qui se confond avec un des grands axes stratégiques de l'Empire, de Rhodes à Constantinople, on ne voit pas en mer Égée de spécialisation qui permette une population dense nourrie depuis ailleurs. Pas d'olivaie généralisée, pas de choix de l'arboriculture, mais des compromis. L'île trop menacée et trop pauvre est abandonnée: on va y chasser, y cueillir les plantes médicinales. De manière analogue, au XVe siècle, les Siciliens vont recueillir l'orseille, plante tinctoriale, sur le littoral des Égades désertes et établissent là des carrières dont les profondeurs servent de refuge aux tailleurs de pierre au passage des galiotes hafsides.

La variété des situations est donc encore plus grande que dans les modèles proposés par les auteurs britanniques, qui ont parfaitement saisi la volatilité des économies insulaires. Les temps changent rapidement, la position stratégique des îles se modifie et les profits que peuvent tirer les insulaires de leur position sur les routes de commerce se dissipent. Une formule de Lucien Febvre oppose les îles-carrefours et les îlesprisons ${ }^{15}$. L'abondance de ces dernières dans le monde byzantin comme dans la Sicile des XIV et $\mathrm{XV}^{\mathrm{e}}$ siècles montre que la connexion entre les îles n'est pas si générale. Ces lieux d'exil, on dira confino, plutôt que camps d'enfermement, ne demandent pas une bien grande surveillance, que les effectifs des polices d'alors seraient incapables d'assurer : leur efficace réside simplement dans l'isolement réel des mondes insulaires. C'est une limite sérieuse à l'option de l'emporium.

Emporium insulaire, réalité et fiction

16 La précieuse étude des îles byzantines montre également que l'emporium insulaire peut être une réalité : au VIII ${ }^{\mathrm{e}}$ siècle les bulles des commerciaires attestent leur présence en Égée, à Milos, à Andros, à Thèra, à Anaphè, à Ios et à Amorgos. Ces fonctionnaires disparaissent évidemment avec l'émirat de l'Égée et l'Arabocratie. Seule, mais à l'ouest et hors de l'Égée, Céphalonie manifeste la continuité des trafics. Au XI ${ }^{\mathrm{e}}$ siècle, Corfou, la Crète, l'Eubée et Rhodes encadrent une mer intérieure encore inerte, mais où Chios et Mitylène reprennent quelque activité d'échange. Le monastère de Patmos se dote alors d'une petite flotte de barques exemptée de taxes. Elle unit les divers domaines dispersés sur les îles voisines de Léros et de Lipsos, puis en Crète, et exporte sans doute une part du surplus. La conjoncture politique et économique a donc joué d'abord, dans 
ce temps mort, contre les îles : l'Empire byzantin s'est ruralisé, continentalisé. On a vu que Pantelleria et Malte, qui ont joué le rôle d'emporium jusqu'au VIII siècle, ont été ensuite totalement abandonnées : le monde de l'Islam est longtemps avant tout terrien, même si quelques relations navales pacifiques se laissent entrevoir avec celui de Byzance. Ce sont des rapports directs qui ne font plus jouer les îles.

Mais la conjoncture a changé et l'activité maritime s'est réveillée, en commençant par les relations à longue distance, au XI $\mathrm{XI}^{\mathrm{e}}$ siècle, temps d'abord de la Geniza, puis des républiques italiennes. Peut-on dire que les îles retrouvent une vocation perdue de relais, une fonction de redistribution? Il faut se garder de figer une image qui était pleinement valable pour l'Antiquité classique, peut-être encore acceptable pour l'Antiquité tardive et la transition, mais limitée à peu de cas pour le Moyen Âge central. Certaines grandes îles ont au contraire perdu ce rôle de transitaire qui subsiste entre $\mathrm{XI}^{\mathrm{e}}$ et $\mathrm{XII}{ }^{\mathrm{e}}$ siècle, à la fin de l'époque fâtimide, pour laquelle nous disposons du témoignage d'al-Makhzûmî : les marchands siciliens et sardes jouissent d'un traitement privilégié dans les ports égyptiens ${ }^{16}$. Mais les petites îles n'ont pas retrouvé la fonction qu'elles gardaient dans l'Antiquité tardive : la géographie d'Idrîsî, attentive pourtant aux phénomènes commerciaux, signale des entrepôts sur les côtes africaines, syriennes et europénnes, Anfâ, Bâdis des Ghumâra, Matifou, Mahdiyya, Sfax, Lebda, à demi abandonnée,Tripoli, Almyros, etc. Mais ce sont des ports continentaux, et non plus des îles.

Les grandes îles seules apparaissent solidement ancrées dans la fonction d'entrepôt de redistribution aux siècles successifs : Majorque joue ainsi avec succès le rôle de portail du Maghreb, favorisée par la proximité du littoral africain. Les marchands majorquins prolongent d'abord, jusqu'en 1246, l'échange intramusulman ${ }^{17}$ pour assurer ensuite la greffe des exportations françaises vers l'Afrique, estanforts d'Arras et de Saint-Omer, bleu de Provins, rayé d'Ypres, etc. vers Oran et Tunis. La neutralité politique du royaume de Majorque garantit l'ouverture œcuménique de son port. Ceuta, quasi-île, est également laissée dans un statut d'autonomie qui évoque le "port of trade » de K. Polyani. Sous l'autorité des Banû 'Azafî, famille de savants et d'armateurs, elle peut donc accueillir les navires de toute origine, sans trop se préoccuper des alliances et des inimitiés du pouvoir marocain ${ }^{18}$. Le port de Chios, sous la domination génoise, a un rôle régional remarquable, mais il ne concurrence pas les grands emporia de l'Orient, Péra et Caffa. Le manuel de Pegolotti, reflet de la réalité commerciale à la fin du XIII ${ }^{e}$ siècle, n'accorde cependant à Ceuta qu'une place très limitée et ignore Chios. Tout son intérêt va aux très grandes îles, Sicile, Sardaigne, à Chypre, à Majorque, et à Rhodes. Seule Nègrepont (Chalcis en Eubée) a des relations avec l'Italie. On ne comptera pas les îles dalmates, Pago, Veglia, ou encore Ibiza/Eivissa, spécialisées dans l'extraction du sel, ni Djerba dont les relations bilatérales se font exclusivement avec la Sicile (blé contre huile).

On sera donc prudent avec la notion d'emporium : selon les époques et selon les sources, elle s'appliquera à des îles plus petites ou seulement à de grands ports de redistribution, enlevant au monde insulaire toute spécificité dans le champ commercial. La vulnérabilité des entrepôts littoraux fait qu'on évite quelquefois, comme en Sicile, d'exposer les marchandises à une attaque navale et qu'on stocke les grains au plus près des productions. Disposer d'un point d'échange off shore n'a d'intérêt que si un accord tacite le protège, comme pour Ceuta.

Les îles et la piété des gens de mer 
La sainteté des îles, comme celle des caps, a été un thème récurrent de l'historiographie et de l'anthropologie méditerranéennes. Elle s'insère dans un courant idéologique particulièrement fort en Italie méridionale: "survie» de l'Antiquité païenne, "paganisme éternel» de Carlo Levi, religion naturelle animiste et contractualiste d'Emilio Sereni, etc. On débouche sur la permanence naturaliste des sites et des cultes, thème critiqué par Horden et Purcell, mais non sans nostalgie pour la théorie de Sir John Frazer et son Rameau d'or. Cette permanence fictive pollue l'histoire locale des lieux saints ; il faut trier, dissocier les époques et les cultures. Patmos a ainsi connu un culte d'Artémis Patmia, puis un monastère dédié à l'Évangéliste, mais ce sanctuaire n'est construit qu'au XI ${ }^{e}$ siècle : il n'y a entre eux aucune continuité.

21 Les temples qui balisent la mer païenne n'ont laissé de traces qu'archéologiques et toponymiques (Cap Colonne, Cap Couronne). Il se maintient seulement dans la culture savante des municipes musulmans et des géographes quelque statue talismanée, comme celle de Cadix, et des "idoles", sanam, simples pierres levées ou colonnes également rappelées par les toponymes, comme à Sélinonte. Elles ne sont objet d'aucune vénération, mais de curiosité et de crainte. L'aménagement en églises des anciens lieux de culte païen est rare, tardif, et ne laisse guère de traces dans les îles.

Deux points, cependant, attirent l'attention. C'est d'abord la localisation de monastères dans les îles, qu'elles soient de nouveaux déserts «égyptiens » décalés dans le temps, comme le Lérins au Ve siècle, la quasi-île sainte de l'Athos au $\mathrm{X}^{\mathrm{e}}$, Gorgona et Patmos au $\mathrm{XI}^{e}$, les Strophades au XIV ${ }^{e}$, ou au contraire des avant-postes de l'Empire et de l'Église, comme Saint-Jean de Pantelleria, prospère au VIII ${ }^{\mathrm{e}}$ siècle, lieu d'exil pour les membres de la hiérarchie épiscopale byzantine, et qui comptait plus de 60 moines en 806, quand un raid de Musulmans d'Espagne a entraîné leur enlèvement et le rachat par Charlemagne de quelques-uns d'entre eux. Cette présence de monastères ne fait nullement de la mer un espace particulièrement saint : dans l'ensemble de l'Égée, il n'y a qu'une dizaine d'îles à recevoir cette protection spirituelle avant le XIII ${ }^{\mathrm{e}}$ siècle. Autour de la Sicile, il n'y en a aucun avant la construction du monastère d'Ustica au XIII , vite suivie d'abandon. Rien à Malte, rien à Pantelleria, d'ailleurs peuplée de Musulmans. Aucun, non plus, dans les îles qui entourent la Sardaigne. Le monastère n'ancre guère la géographie insulaire dans celle de la mobilité, ni dans celle des commerces fixés par les foires. Le survol des lieux de pèlerinage répertoriés par Pierre Maraval pour la fin de l'Antiquité et le haut Moyen Âge oriental ${ }^{19}$ et par Élisabeth Malamut pour la haute époque byzantine ${ }^{20}$ ne laisse percevoir que quelques sites insulaires : quelques lieux saints à Chypre (saint Barnabé, saint Épiphane, le martyr Tychon, les évêques Héraclide, Triphyllios, Auxibios, Théodote), saint Isidore et sainte Myrope à Chios, saint Clément dans l'île de Cherson, saint Pancrace à Taormine, auxquels il faut ajouter saint Gui, à Capo San Vito de Sicile, attesté à l'époque musulmane. saint Tite, saint Cyrille et l'évêque Myron en Crête. On voit que ce sont presque uniquement des sanctuaires de grandes îles, disposant d'évêchés, espaces quasi-continentaux. Rien donc qui ressemble à un maillage étroit. Ce sont les côtes qui, comme l'a noté Christophe Picard, opposent, unissent ou alternent monastères et ribâts dans un souci commun de protection ${ }^{21}$. Plus tard, au XII siècle, les reliques de Barthélemy sanctifieront Lipari, au seuil des portes de l'enfer, Vulcano et Stromboli, mais l'île de Vulcano est déjà gardée par des ermites qui comptabilisent les grands personnages entraînés dans ses profondeurs. 

navigateurs et des voyageurs à la mer et de manière plus œecuménique encore: les récits de voyages ${ }^{22}$ attestent la présence d'ermites le long de la route des pèlerinages chrétiens, de Venise à la Palestine, à Saint-André, près de Lissa, sur l'île de Sapienza, près de Modon, à l'Escandeye/Dia, dans la mer de Candie. Ils associent dans la même vénération les franciscains observants de Santa Maria delle Grazie devant Lesina, les observants qui vivent sur un écueil devant Curzola (Otok aujourd'hui), les «basiliens " des Strophades et ils évoquent encore les calogers du monastère des Chats au « chief de Guavata » de Chypre (cap Gata). Les pèlerins et voyageurs musulmans attestent aussi la présence d'ermites sur les îles : Ibn Battûta visite un couvent et y rencontre un vieillard au Mont Lu'mân (île Hallaniya) sur les côtes du Dhafâr face à l'Océan indien ${ }^{23}$. Ce sont d'ailleurs les patrons de l'érémitisme, Élie et Khidr, qui protègent les derviches d'Abbâdân, à l'embouchure du Chatt al-Arab, et ceux du détroit d'Hormûz, et encore l'ermitage de la montagne qui s'avance devant Sinope, sur la Mer noire, comme Moïse et Khidr défendent et bénissent la quasi-île de Ceuta. Spécialistes des eschatai, des passages lointains, saints et ermites balisent les limites d'un monde dangereux, dont les îles ne sont qu'un élément.

Il resterait à voir en quoi, au Moyen Âge, les îles participent à la circulation des objets de la piété, et pas seulement de la dévotion des gens de mer. Sont-elles des étapes dans la diffusion du culte des saints ou de nouvelles formes de dévotion ? Il est difficile de le préciser: seule Ceuta, dont le statut d'île est seulement métaphorique, apparaît clairement comme le portail par lequel entrent au Maghreb extrême les nouveautés orientales. Premier mawlid du prophète vers 1260 , lié à la première affirmation des pauvres par choix, première medersa, dès 1238 , première cité où les nobles descendants de 'Alî, les shurafâ', accèdent au pouvoir, dès le $\mathrm{XI}^{\mathrm{e}}$ siècle. Mais il semble bien que ce cas soit unique et rien de semblable, aucun portail, ne se dessine sous d'autres cieux.

Les îles et le pouvoir naval théoricien sulfureux sur d'autres terrains, Carl Schmitt ${ }^{24}$, a l'avantage de faire ressentir l'opposition entre les îles passives et les îles actives. Ces dernières ne subissent pas la domination venue de la mer, mais s'arment pour assurer leur défense ou pour mettre sur pied une domination régionale ou même une thalassocratie ${ }^{25}$. Le modèle invoqué était l'Angleterre d'Élisabeth Ire, qui marie course, armement commercial et capacités militaires. La Sicile des derniers Aghlabides et des émirs kalbites, au $\mathrm{X}^{\mathrm{e}}$ et au début du $\mathrm{XI}^{\mathrm{e}}$ siècle retrouve cette figure, à une échelle modeste, mais avec un acharnement remarquable : la guerre de course est menée contre la Calabre et la Pouille $(975,1002$, 1009, 1016, 1031), mais aussi contre Alméria (954) et même contre Tripoli et Sfax, ravagée en 915. On retrouve les caractéristiques de l'Arabocratie dans l'Archipel: la flotte des Rabadîs de Séville, basée d'abord en 815 à Alexandrie, puis en Crète à partir de 827 et à Athènes constitue une zone de pillage et de réduction en esclavage qui s'étend à toute l'Égée, jusqu'aux premiers succès de Romain Lécapène (934 à Mitylène) et à la reconquête de la Crète en 961. La guerre n'exclut pas le trafic, mais la principale marchandise est l'homme.

La Sicile normande offre une autre configuration du pouvoir naval, héritée de Byzance : un lourd appareil fiscal et des mobilisations de rameurs pèsent sur les communautés rurales, qui doivent aussi fournir le bois aux arsenaux. Faute de marine de commerce active, c'est l'État qui se charge de tout et qui choisit l'insularité : la capitale est à l'abri 
derrière le double glacis de la Terreferme et d'une mer dominée, non plus partagée, mais divisée. Les Génois sont exclus de l'espace qui va de la Sicile au littoral maghrébin vite conquis par la flotte de l'émir Georges d'Antioche entre 1142 et 1148. Et la flotte sicilienne peut aller frapper très loin: Nègrepont, Damiette, Alexandrie, Majorque, Thessalonique, Tyr. Elle fait alors, jusqu'en 1189, jeu égal avec Pise et Gênes et permet à l'État sicilien d'appliquer pendant quelque temps une stratégie de conquête des rives opposées des mers étroites, Albanie, Dalmatie, Ifrîqiyya. De nouveau, de 1282 à 1350, un effort fiscal extrême permettra à l'insularité sicilienne, choisie et liée à la Révolution des Vêpres et au changement dynastique, de lever une flotte de galères capable de faire face à la puissance angevine soutenue par le grand arrière français. La Sicile aragonaise en profitera pour reprendre pied à Djerba, perdue vers 1150 .

Passé 1350, la Sicile reviendra à la position d'île passive, qu'elle partage avec une Sardaigne à l'inertie pérenne depuis l'époque byzantine et exposée aux conquêtes. La Chypre byzantine n'exploite que fugitivement sa position géographique parfaite et, sous les Lusignan, l'île ne fait qu'accueillir les corsaires catalans, entraînant les représailles sévères des Mamlouks. L'autonomie et une relation de service caractérisent la Majorque des Banû Ghâniyya sous le pouvoir lointain des Almoravides : leur arsenal et leur flotte couvrent la frontière maritime vers l'Europe et l'Orient. Le choix d'être une île, avec ses risques et les profits qu'on en attend, est ici caractérisé. En 1127, après le sac de la ville de Majorque par l'expédition pisane et génoise, le nouveau gouverneur almoravide veut la faire reconstruire à l'intérieur des terres. La population se révolte et obtient le remplacement du gouverneur par Ibn Ghâniyya qui maintient la capitale sur la mer. Majorque n'use cependant de sa situation géographique exceptionnelle dans le champ politique que quand les Banû Ghâniyya transforment l'archipel en base de reconquête du Maghreb. Ils débarquent sans opposition à Bougie en 1184, et soulèvent les Arabes contre la domination almohade, puis se replient sur Tozeur et, de concert avec l'envoyé de Saladin Qarâqûsh et ses troupes turques, leurs partisans ravagent l'Afrique du nord jusqu'en 1237, pendant plus de cinquante ans.

Les pouvoirs navals proprement insulaires sont, on le voit, peu nombreux, pour ne pas parler de thalassocratie: la seule ambition d'une domination navale étendue à la Méditerranée centrale, Tyrrhénienne, Canal d'Afrique, Ionienne et Adriatique, est celle de la dynastie normande de Sicile, et encore s'agit-il toujours de mers partagées, à l'exception de la mer d'Afrique. À un niveau d'organisation et de forces plus modeste, les îles peuvent jouer encore un rôle autonome, dans l'ombre d'un pouvoir plus vaste, ou n'être qu'un rouage dans une stratégie d'ensemble, un préside. Ceuta, que nous assimilons toujours à une île, dispose d'un arsenal, d'une position remarquable, de ports convenables, et, dès le $\mathrm{XI}^{\mathrm{e}}$ siècle, elle devient la base de la tentative des Hammûdites de fonder sur le Détroit un califat 'alide. Trop faible pour assurer le triomphe de ce projet, elle passe ensuite à une autonomie étendue sous Saqût alBarghawâtî de 1041 à 1073, et presque continûment de 1234 à 1329, avec des périodes intermédiaires de contrôle plus étroit des Almoravides et des Almohades. La flotte assure la quasi-indépendance de la république oligarchique, puis de la Seigneurie, à l'italienne, et assume des missions de guerre pour le compte de l'Empire continental marocain ou d'imprécises, mais probables, actions de course.

Le roi de la taifa de Dénia, Mudjâhid, avait déjà, en 1014-1015, tenté de fonder un empire maritime par la conquête réussie de Majorque, puis par celle de la Sardaigne, manquée. La souplesse des relations entre centre impérial et périphérie dans le monde 
musulman permet ces relations complexes entre des pouvoirs dont la taille n'est pas trop dissemblable. Dans l'ensemble des pays chrétiens, au contraire, les périphéries corsaires, îles ou ports isolés, sont de petit format et la course tâche de distinguer entre port d'armement et port de recel, pour éviter représailles et enquêtes. Seul, l'État des Hospitaliers dispose d'un vrai centre corsaire qui coïncide avec sa capitale.

Mais, comme dans le monde musulman, la course peut nourrir l'armée navale du pouvoir impérial. On l'a observé dans le monde catalan au XIV siècle: il dispose d'avant-postes en Sardaigne, à Cagliari et à Alghero, en Sicile, à Syracuse et à Augusta, dans l'Archipel, à Famagouste, d'où des enfants perdus peuvent assez librement harceler les lignes de navigation génoises et vénitiennes. Avec l'introduction des marines atlantiques, au $\mathrm{XV}^{\mathrm{e}}$ siècle, Basques, Cantabriques et Portugais se basent à Ceuta, à Malte, à Pantelleria; ils servent l'Empire d'Alphonse V d'Aragon, sans lui coûter un florin. Les prises corsaires sont censées financer la formation d'une flotte souple et l'entretien des châteaux. Malte et Pantelleria montrent que la réalité est souvent décevante et que le coût retombe sur les habitants de ces bases.

31 L'Empire a le souci en effet, dans la tradition byzantine et vénitienne, relayée par les Hospitaliers de Rhodes, d'assurer des bases navales, des présides, le long des littoraux, et, si possible, à l'abri d'un bras de mer. Rab/Arbe, en Dalmatie, Corfou, Djerba ont déjà servi de points d'appui avancés à la flotte normande; dans les projets d'Alphonse $\mathrm{V}$ d'Aragon, les présides continentaux, comme Bernik (Benghazi) en Cyrénaïque, Castiglione della Pescaia en Toscane, alternent avec les îles. Castellorizo est ainsi mise par les Hospitaliers au service de la flotte impériale de l'amiral Bernat de Vilamarí et le prince aragonais avait le dessein de fortifier Zembra.

Un rêve de grand empire naval qui enserre le Maghreb d'une "connectivité " pour le moins hostile est celui que forge l'infant don Luis de la Cerda, amiral de Philippe VI, et qu'il fait accepter par Clément VI en 1344 : «Prince de la Fortune », il est investi par le pape de l'archipel des Canaries à peine exploré. Son royaume des Îles fortunées comprendra cependant aussi l'île méditerranéenne de la Galite qu'il lui reste à occuper et à munir d'une forteresse, comme le reste de ses États. Le projet était destiné à constituer une belle seigneurie corsaire, ou à rester un Empire imaginaire.

On peut finir sur ce point: les îles méditerranéennes présentent des réseaux exploitables, économiquement et militairement, mais le Moyen Âge central et tardif a utilisé imparfaitement ces potentialités. Les grands trafics de masse dominent largement et interdisent de surestimer ces relations moyennes entre ports d'importance secondaire et qui utilisent les outils du cabotage. Les îles sous-tendent également une part importante de l'imaginaire du monde maritime: du communisme liparéen antique à l'accession des esclaves maltais au service militaire, au mariage et à la citoyenneté, et jusqu'à l'élaboration de grands projets de royautés Outre-mer, précoces mais réalistes, elles alimentent des réflexions politiques qui ne sont pas négligeables. Mais c'est par leur étrangeté, plutôt que par la familiarité qui naît de leur présence dans le tissu connectif. Rappelons que les portulans les ont mises longtemps à part, comme un autre monde, ce que les insulaires postérieurs reprendront encore au $\mathrm{XVI}^{\mathrm{e}}$ siècle. Un halo de mystère les enveloppe, qui correspond sans doute à beaucoup de crainte et à un éloignement persistant. 


\section{NOTES}

1. The Corrupting Sea. A Study of Mediterranean History, Oxford, 2000.

2. La Méditerranée et le monde méditerranéen à l'époque de Philippe II, Paris, 1996 2.

3. "Challenging Braudel : a new vision of the Mediterranean ", Journal of Roman Archoeology, 14, 2001, p. 419-453.

4. La Population des îles grecques : essai de géographie insulaire en Méditerranée, Aix-enProvence, 1974.

5. E. Ch. SEMPLE, The Geography of the Mediterranean Region. Its relation to ancient history, Londres, 1932.

6. Je renverrai à mes articles, " Pantelleria entre l'Islam et chrétienté », Cahiers de Tunisie, XIX, 1971, p. 105-127 ; «Sicile, Malte et monde musulman », dans Malta, a Case Study in International Cross-Currents (Malte, déc. 1989), S. Fiorini et V. Mallia-Milanes éd., Malte, 1991, p. 47-79 ; « Malte et l'Afrique (1282-1492) », Le Carrefour maltais, Revue du Monde Musulman et de la Méditerranée, 71, 1994, p. 63-73 ; « Genèse de l'identité maltaise ", dans Mutations d'identité en Méditerranée. Moyen Âge, époque contemporaine, Paris, 2000, H. Bresc et C. Veauvy éd., p. 141-157 ; « Du ribât au presidio, les enjeux et les contrôles des Détroits siciliens ", dans Controllo degli Stretti e insediamenti militari nel Mediterraneo (colloque de Spolète, 3-4 sept. 1997), Rome, 2002, p. 97-127.

7. Le Voyage d'Outremer en Jherusalem de Nompar, seigneur de Caumont, éd. P. NOBLE, Oxford, 1975.

8. J. M. BRINCAT, Malta 870-1054. Al-Himyarî's Account and its Linguistic Implications, La Valette, 1995.

9. L'île Saint-Nicolas, devant Parenzo/Porec, « sert pour radrechier les mareniers à entrer au port dudit Parence » (Voyage de Georges Langherand, éd. Marquis de Godefroy Méniglaise, Mons, 1861 : au 9 juin 1486).

10. Idrîsî, La Première géographie de l'Occident, H. Bresc et A. Nef prés., Paris, 1999.

11. Le Victorial. Chronique de don Pero Niño, comte de Buelna (1378-1453) par Gutierre Díaz de Gamez, son porte-bannière, trad. et prés. J. Gautier Dalché, Turnhout, 2001.

12. Ludolphe de Suchem, De Itinere Terrce sanctæe, éd. F. Deycks, Stuttgart, 1851, p. 26 : de die cum fumo et de nocte cum flamma.

13. Ch.-E. Dufourcq, Recueil de documents concernant les relations des pays de la Couronne d'Aragon avec le Maghreb de 1212 à 1323, thèse complémentaire de doctorat d'État, Paris, 1965, dactylographié, p. 509, nº 654.

14. É. Malamut, Les Îles de l'Empire byzantin (VII ${ }^{\mathrm{e}}$-XII ${ }^{\mathrm{e}}$ siècles), Paris, 1988.

15. L. Febvre, La Terre et l'évolution humaine, Paris, 1922, p. 248.

16. C. Cahen, Makhzûmiyyât : études sur l'histoire économique et financière de l'Égypte médiévale, Leyde, 1977.

17. D. ABULAFIA, A Mediterranean emporium. The Catalan kingdom of Majorca, Cambridge, 1994, p. 122 : exportation de tissus de lin de Xativa à Ceuta en 1242 et 1246.

18. H. Ferhat, Sabta : des origines au XIV siècle, Rabat, 1993.

19. Lieux saints et pèlerinages d'Orient. Histoire et géographie. Des origines à la conquête arabe, Paris, 1985.

20. Sur la route des saints byzantins, Paris, 1993.

21. C. Picard, L'Océan Atlantique musulman. De la conquête arabe à l'époque almohade, Paris, 1997, p. 82-90. 
22. En particulier Nompar de Caumont (1420) ; Pero Tafur, Andanças e Viajes (1435-1439), éd. M. Jiménez de la Espada, Madrid, 1874 ; Santo Brasca, Viaggio in Terra santa, éd.

A. L. Momigliano Lepschy, Milan, 1966 (1480) ; Voyage de Jacques Le Saige de Douai à Rome, Notre-Dame de Lorette, Venise, Jérusalem, éd. H. R. Duthillœul, Douai, 1851 (1518).

23. Ibn Battûta, Voyages, trad. C. Defremery et B. R. Sanguinetti, Paris, 1982, t. II, p. 106.

24. Terre et mer, Paris, 1985 , p. 77, citant Shakespeare : « Bijou serti d'une mer d'argent / Qui lui est un rempart, / Un fossé qui défend la maison ».

25. On accepte le concept, au sens où l'emploie Hélène Ahrweiler (Byzance et la mer. La marine de guerre, la politique et les institutions maritimes de Byzance aux VII ${ }^{e}-\mathrm{XV}^{e}$ siècles, Paris, 1966), pour en critiquer le coût et la dimension chimérique.

\section{AUTEUR}

\section{HENRI BRESC}

Université Paris X-Nanterre, Département d'Histoire, 200, avenue de la République, F-92000 Nanterre Cedex 